\title{
Erratum to: Which nomogram is best for predicting non-sentinel lymph node metastasis in breast cancer patients? A meta-analysis
}

\author{
Liling Zhu $\cdot$ Liang Jin $\cdot$ Shunrong Li $\cdot$ \\ Kai Chen · Weijuan Jia • Quanyuan Shan • \\ Stephen Walter $\cdot$ Erwei Song $\cdot$ Fengxi Su
}

Published online: 17 April 2013

(C) Springer Science+Business Media New York 2013

Erratum to: Breast Cancer Res Treat (2013)

137:783-795

DOI 10.1007/s10549-012-2360-6

Unfortunately, in the original publication of the article, there were several typos and errors in the text, figures and tables. The authors apologize for these errors. The corrected content is given below. The correct Table 1 of Electronic Supplementary Material is also provided in the link below.

1. Abstract: "To present a systemic..." should be "To present a systematic ..."

2. Introduction (P2, Line1), "The May model..." should be "The Mayo model..."

The online version of the original article can be found under doi:10.1007/s10549-012-2360-6.

Electronic supplementary material The online version of this article (doi:10.1007/s10549-013-2512-3) contains supplementary material, which is available to authorized users.

L. Zhu $\cdot$ L. Jin $\cdot$ S. Li $\cdot$ K. Chen $\cdot$ W. Jia $\cdot$ Q. Shan

E. Song $(\bowtie) \cdot$ F. Su $(\bowtie)$

Department of Breast Surgery, Breast Tumor Center,

Sun Yat-sen Memorial Hospital, Sun Yat-sen University,

Guangzhou, China

e-mail: songerwei02@yahoo.com.cn

F. Su

e-mail: fengxisu@vip.163.com

S. Walter

Department of Clinical Epidemiology and Biostatistics,

McMaster University, Hamilton, ON, Canada
3. Method (P2, 2nd and 3rd paragraph), "(()((non-sentinel [Title/Abstract]) OR non-sentinel[Title/Abstract])..." should be "(((((non-sentinel[Title/Abstract]) OR nonsentinel[Title/Abstract])...”

4. Results, third line: "and 35 studies were included..." should be "and studies from 35 references were included..."

5. In Fig. 1, the number "155" should be "146".

6. In Figs. 2, 4, 6a and 7, the corrected data were incorporated and given below. 
Fig. 2 Forest plots of the

Cambridge model validated in eight studies

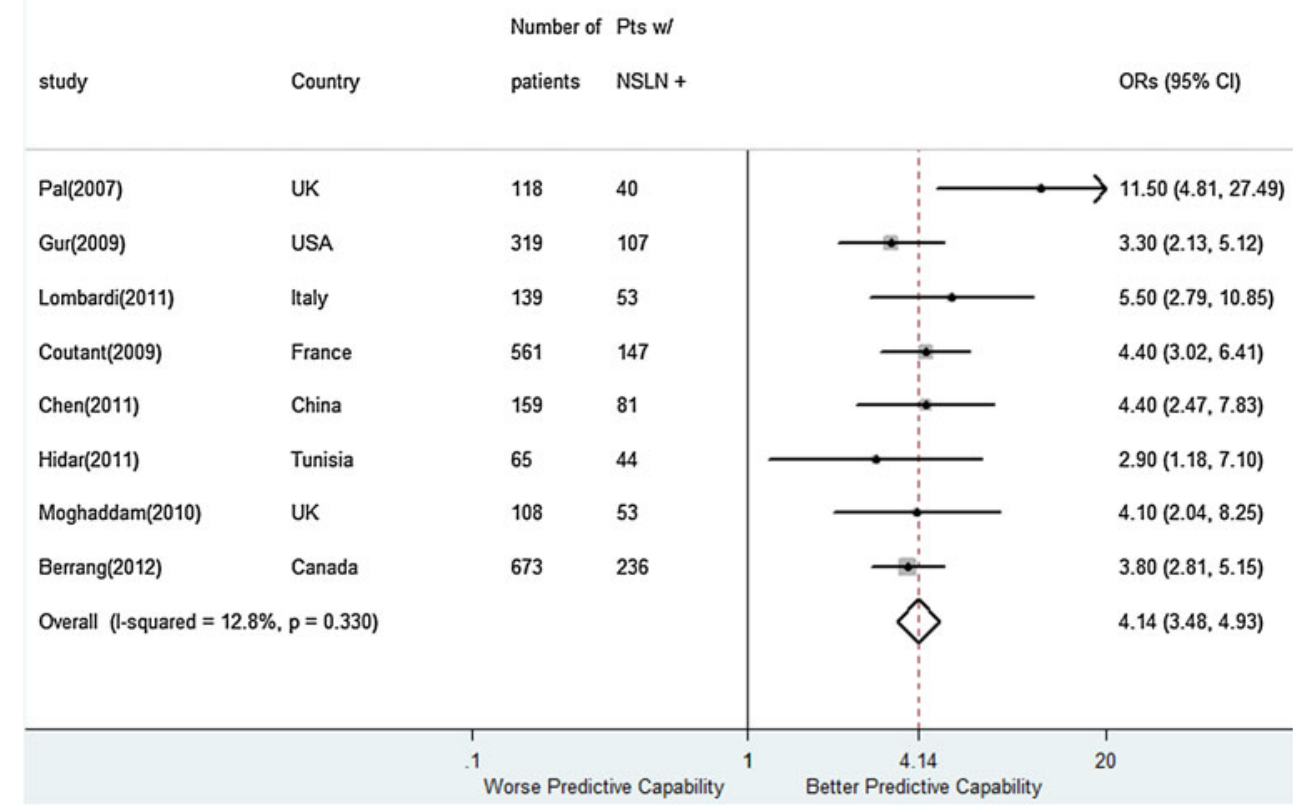




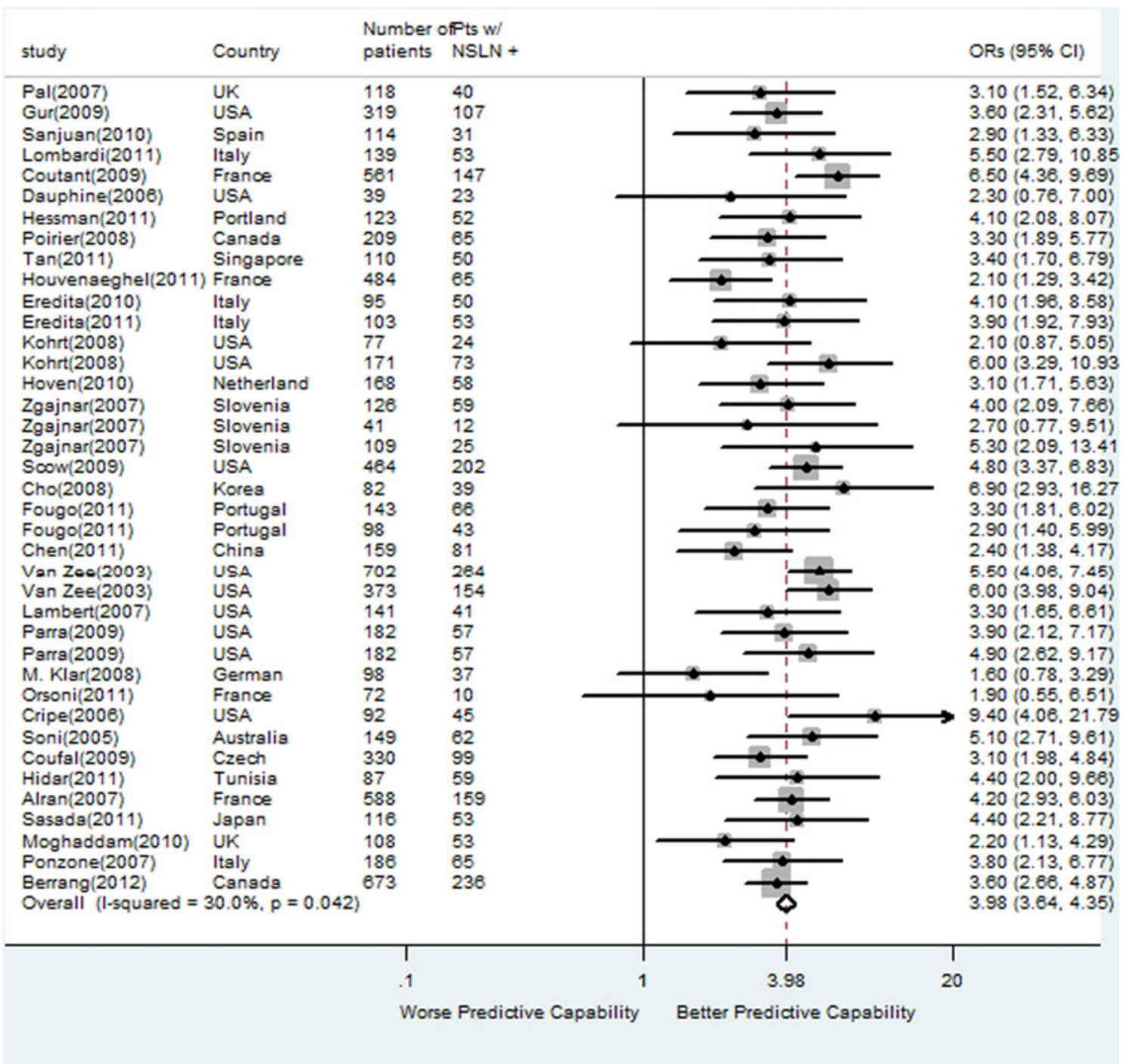

Fig. 4 Forest plots of the MSKCC model validated in 39 studies 
Fig. 6 Forest plots of the

Tenon model validated in a 14 studies. The Berrang (2012)

study has been added into the subgroup analysis
A

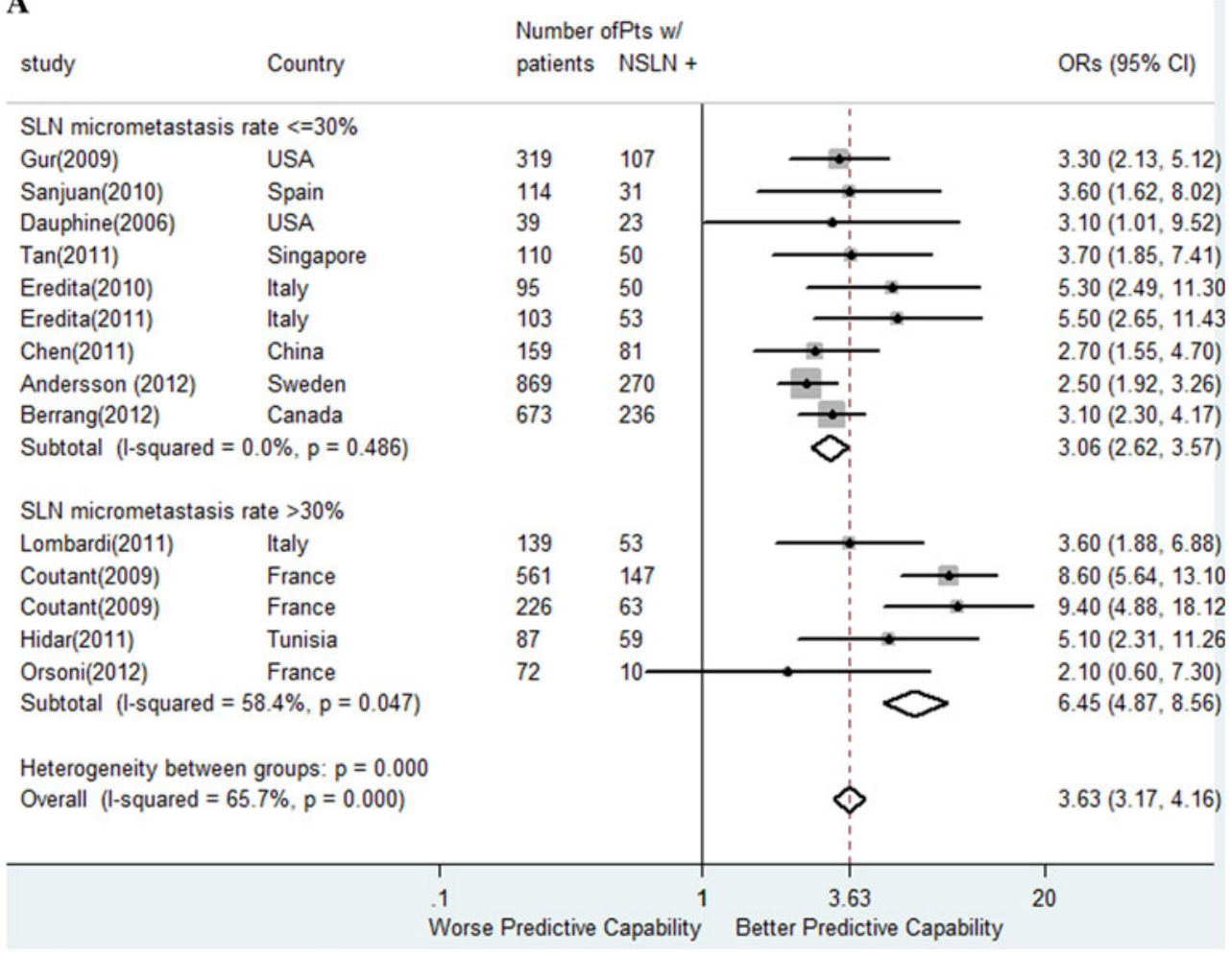


Fig. 7 Forest plots of the Stanford model validated in a 12 and b 14 studies. The Berrang (2012) study has been added into the subgroup analysis
A

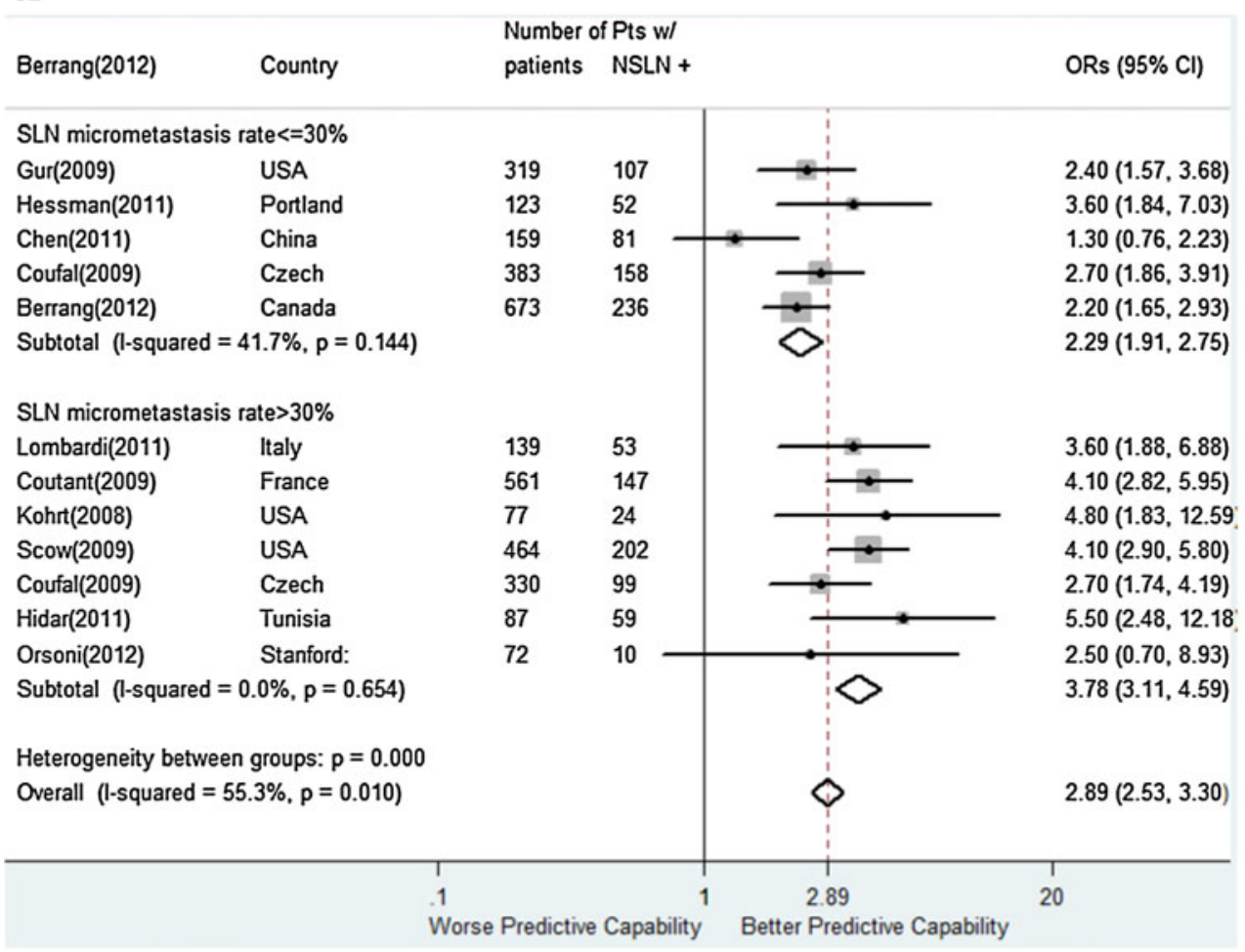

B

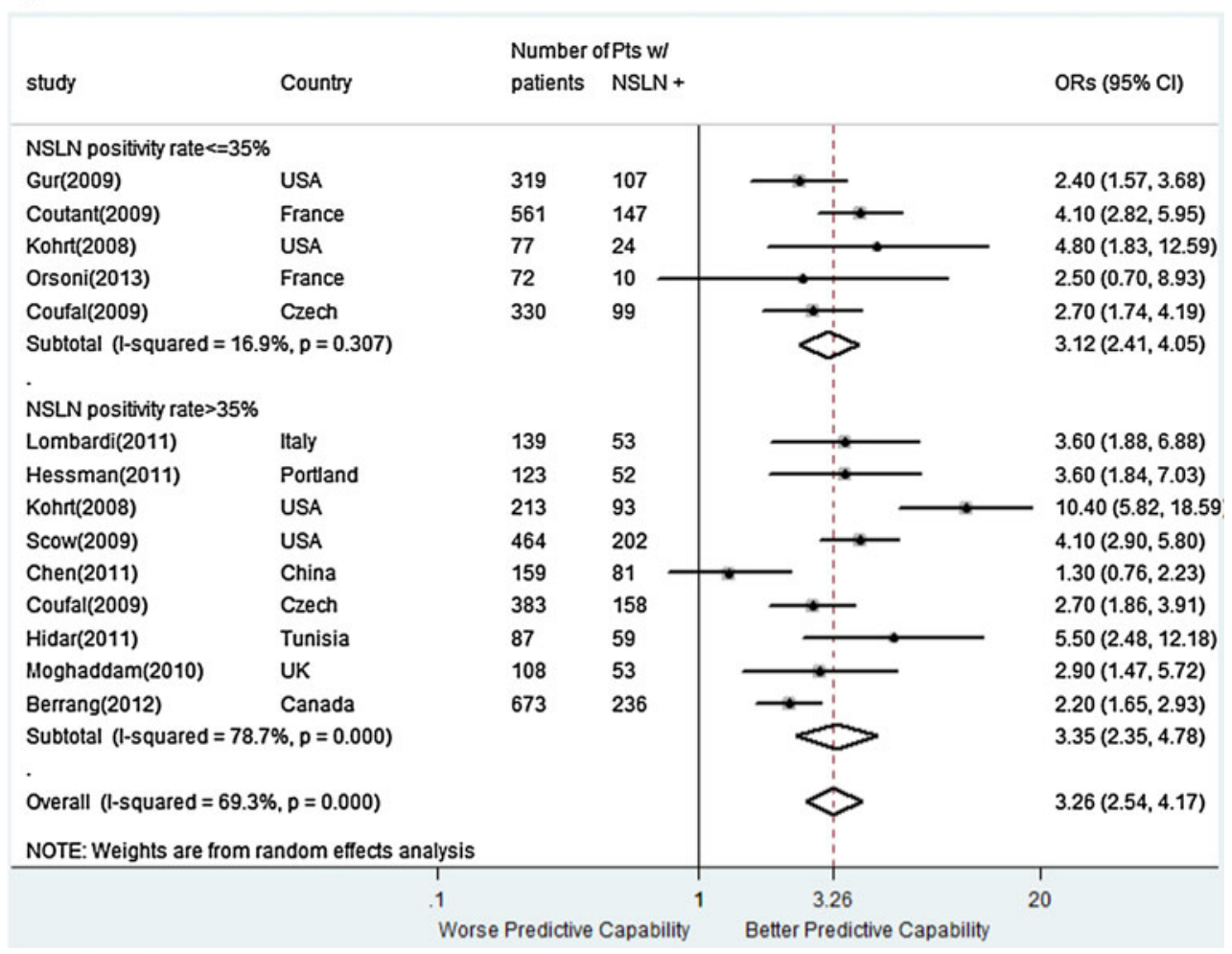


Table 2 Summary of the pooled ORs and their corresponding AUCs of each models

\begin{tabular}{|c|c|c|c|c|c|c|c|c|c|}
\hline \multirow[t]{2}{*}{ Models } & \multirow{2}{*}{$\begin{array}{l}\text { No. of } \\
\text { studies }\end{array}$} & \multicolumn{3}{|c|}{ Heterogeneity test } & \multicolumn{2}{|c|}{ Combined odds ratio } & \multicolumn{2}{|c|}{ Converted AUC } & \multirow{2}{*}{$\begin{array}{l}\text { Heterogeneity } \\
\text { between } \\
\text { subgroups, } P\end{array}$} \\
\hline & & $x^{2}$ & df & $P$ & OR & $95 \% \mathrm{CI}$ & AUC & $95 \% \mathrm{CI}$ & \\
\hline Cambridge & 8 & 8.03 & 7 & 0.33 & 4.14 & $3.48-4.93$ & 0.72 & $0.70-0.75$ & \\
\hline MDA & 4 & 2.14 & 3 & 0.54 & 3.69 & $2.79-4.89$ & 0.71 & $0.67-0.74$ & \\
\hline MSKCC & 39 & 54.32 & 38 & 0.04 & 3.98 & $3.64-4.35$ & 0.72 & $0.70-0.73$ & \\
\hline Mayo & 6 & 15.12 & 5 & 0.01 & 4.33 & $3.22-5.82$ & 0.73 & $0.69-0.77$ & \\
\hline Mayo without Berrang (2012) & 5 & 4.24 & 4 & 0.26 & 5.10 & $4.21-6.18$ & 0.75 & $0.72-0.77$ & \\
\hline Tenon & 15 & 38.50 & 14 & $<0.01$ & 4.07 & $3.17-5.21$ & 0.72 & $0.68-0.75$ & \\
\hline \multicolumn{10}{|l|}{ Subgroup analysis } \\
\hline NSLNs positivity rate $\leq 35 \%$ & 6 & 32.59 & 5 & $<0.01$ & 4.30 & $2.51-7.39$ & 0.73 & $0.65-0.79$ & 0.74 \\
\hline NSLNs positivity rate $>35 \%$ & 9 & 5.80 & 8 & 0.67 & 3.58 & $2.95-4.34$ & 0.70 & $0.67-0.73$ & \\
\hline Tenon & 14 & 37.86 & 13 & $<0.01$ & 3.63 & $3.17-4.16$ & 0.70 & $0.68-0.72$ & \\
\hline \multicolumn{10}{|l|}{ Subgroup analysis } \\
\hline SLN micrometastasis rate $\leq 30 \%$ & 9 & 7.48 & 8 & 0.49 & 3.06 & $2.62-3.57$ & 0.68 & $0.66-0.70$ & $<0.01$ \\
\hline SLN micrometastasis rate $>30 \%$ & 5 & 9.62 & 4 & 0.05 & 6.45 & $4.87-8.56$ & 0.78 & $0.74-0.81$ & \\
\hline Stanford & 14 & 42.39 & 13 & $<0.01$ & 3.26 & $2.54-4.17$ & 0.69 & $0.65-0.72$ & \\
\hline \multicolumn{10}{|l|}{ Subgroup analysis } \\
\hline NSLNs positivity rate $\leq 35 \%$ & 5 & 4.81 & 4 & 0.31 & 3.12 & $2.41-4.05$ & 0.68 & $0.64-0.72$ & 0.83 \\
\hline NSLNs positivity rate $>35 \%$ & 9 & 37.53 & 8 & $<0.01$ & 3.35 & $2.35-4.78$ & 0.69 & $0.64-0.74$ & \\
\hline Stanford & 12 & 24.62 & 11 & 0.01 & 2.89 & $2.53-3.3$ & 0.67 & $0.65-0.69$ & \\
\hline \multicolumn{10}{|l|}{ Subgroup analysis } \\
\hline SLN micrometastasis rate $\leq 30 \%$ & 5 & 6.86 & 4 & 0.14 & 2.29 & $1.93-2.75$ & 0.64 & $0.61-0.66$ & $<0.01$ \\
\hline SLN micrometastasis rate $>30 \%$ & 7 & 4.17 & 6 & 0.654 & 3.78 & $3.11-4.59$ & 0.71 & $0.68-0.74$ & \\
\hline
\end{tabular}

$S L N$ sentinel lymph node, $A U C$ area under the curve, $C I$ confidential interval, $d f$ degree of freedom

Table. 3 Meta-regression for Tenon and Stanford models

\begin{tabular}{llllrrr}
\hline Model & No. of studies & Covariate factors & Exp (b) & Std. Err. & $P$ & $95 \%$ CI \\
\hline Tenon & 14 & SLNs tumor burden (Micrometastasis rate $>30 \%$ vs. $\leq 30 \%$ ) & 1.91 & 0.39 & $<0.01$ & $1.22-2.99$ \\
& 15 & NSLNs tumor burden (NSLN positivity rate $>35 \%$ vs. $\leq 35 \%)$ & 0.90 & 0.23 & 0.68 & $0.52-1.56$ \\
\multirow{2}{*}{ Stanford } & 12 & SLNs tumor burden (Micrometastasis rate $>30 \%$ vs. $\leq 30 \%)$ & 1.65 & 0.23 & $<0.01$ & $1.20-2.26$ \\
& 14 & NSLNs tumor burden (NSLN positivity rate $>35 \%$ vs. $\leq 35 \%)$ & 1.06 & 0.31 & 0.84 & $0.56-2.02$ \\
\hline
\end{tabular}

